\title{
A twelve-wavelength Thulium-doped fibre laser based on a microfibre coil resonator incorporating black phosphorus
}

Shi Li

Harbin Engineering University

Yu Yin

Harbin Engineering University

Elfed Lewis

University of Limerick

See next page for additional authors

Follow this and additional works at: https://arrow.tudublin.ie/prcart

Part of the Electrical and Computer Engineering Commons, Engineering Physics Commons, and the Optics Commons

\section{Recommended Citation}

Shi Li, Yu Yin, Elfed Lewis, Gerald Garrell, Pengfei Wang, A twelve-wavelength Thulium-doped fibre laser based on a microfibre coil resonator incorporating black phosphorus, Optics Communications, Volume 437, 2019, Pages 342-345, ISSN 0030-4018, DOI: 10.1016/j.optcom.2018.12.075.

This Article is brought to you for free and open access by the Photonics Research Centre at ARROW@TU Dublin. It has been accepted for inclusion in Articles by an authorized administrator of ARROW@TU Dublin. For more information, please contact arrow.admin@tudublin.ie, aisling.coyne@tudublin.ie,gerard.connolly@tudublin.ie. Funder: National Key R\&D Program of China; National Natural Science Foundation of China (NSFC); Harbin Engineering University

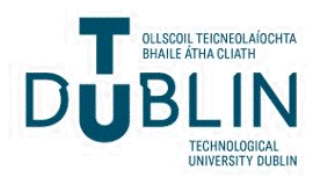


Authors

Shi Li, Yu Yin, Elfed Lewis, Gerald Farrell, and Pengfei Wang

This article is available at ARROW@TU Dublin: https://arrow.tudublin.ie/prcart/19 


\title{
A twelve-wavelength Thulium-doped fibre laser based on a microfibre coil resonator incorporating black phosphorus
}

\author{
Shi Li ${ }^{a}$, Yu Yin ${ }^{a}$, Elfed Lewis ${ }^{b}$, Gerald Garrell ${ }^{c}$, Pengfei Wang a,d,* \\ ${ }^{a}$ Key Laboratory of In-fiber Integrated Optics of Ministry of Education, College of Science, Harbin Engineering University, Harbin 150001, China \\ ${ }^{\mathrm{b}}$ Optical Fibre Sensors Research Centre, Department of Electronic and Computer Engineering, University of Limerick, Limerick, Ireland \\ ${ }^{\mathrm{c}}$ Photonics Research Centre, Dublin Institute of Technology, Kevin Street, Dublin 8, Ireland \\ ${ }^{\mathrm{d}}$ Key Laboratory of Optoelectronic Devices and Systems of Ministry of Education and Guangdong Province, College of Optoelectronic Engineering, Shenzhen \\ University, Shenzhen, 518060, China
}

\section{A R T I C L E I N F O}

\section{MSC:}

$00-01$

99-00

Keywords:

Nonlinear optics

Black phosphorus

Fibre laser

Microfibre coil resonator

\begin{abstract}
A B S T R A C T
A novel multi-wavelength continuous Thulium-doped fibre laser incorporating a microfibre coil resonator based on black phosphorus (MCR-BP) material has been successfully fabricated and demonstrated. A twelve-wavelength spectrum with $0.54 \mathrm{~nm}$ channel spacing has been achieved by simply adjusting the pump source power. A single peak extinction ratio of more than $40 \mathrm{~dB}$ was observed. The superior performance of the MCR-BP described in this article compared to conventional MCR based fibre lasers can be attributed the inclusion of the Black Phosphorous Material. The enhanced lasing is primarily due to the twin properties of the MCR-BP device combining a comblike filter effect and a four-wave-mixing (FWM) effect caused by the nonlinearity of black phosphorus. The experimental results indicate that the novel device can be successfully used as a comb filter and an FWM source to realize a multi-wavelength continuous fibre laser due to the low cost and ease of fabrication of the MCR-BP device.
\end{abstract}

\section{Introduction}

Multi-wavelength fibre lasers have attracted significant recent research attention due to their wide range of applications in laser ranging, distributed multiplexing, spectral analysis, etc [1]. Furthermore, $2 \mu \mathrm{m}$ wavelength fibre lasers have been successfully developed over the last decade due to their inherent advantages of being eye safe and providing low loss atmospheric transmission, therefore making them suitable for use in a number of potential applications including gas sensing, optical communication systems etc. [1,2]. A few techniques have recently been proposed to implement multi-wavelength operation around $2 \mu \mathrm{m}$. Nonlinear devices have previously helped to improve some excellent lasing performance with outstanding potential including provision of compact size, low cost, safety, and have demonstrated effects including nonlinear polarization rotation [3], four-wave-mixing (FWM) [4], Sagnac loop [5], fibre gating [6]. It is widely believed that the four-wave-mixing (FWM) method is an excellent solution for implementing multi-wavelength lasers due to the relatively simple construction of the devices and hence ease of fabrication. Specifically, the FWM method has the significant advantage in that it can excite a stable multi-wavelength output even in a relatively cheap but high-performance two-dimensional (2D) material. Graphene is a typical source of FWM among the numerous 2D materials due to its known high third-order nonlinearity which is desirable for the generation of a stable multi-wavelength output [7]. More recently, researchers have studied new materials with higher nonlinearity and higher damage threshold to improve the performance of continuous wave multi-wavelength fibre lasers. In recent years, black phosphorus has attracted much attention in the lasing field since it can be used a saturable absorber and it possesses ultrahigh nonlinearity $[8,9]$. The excellent nonlinearity is a key factor and it has been established that a small segment of black phosphorus of only several micrometres thickness offers a level of FWM effect in a fibre laser that would otherwise require hundreds of metres of dispersion shifted fibres. Therefore, black phosphorus is an ideal candidate material to generate stable multiwavelength emission around $2 \mu \mathrm{m}$ in fibre lasers.

Microfibre has previously been widely used to fabricate numerous photonic devices since it was initially demonstrated [10]. Researchers have since developed several resonators based on microfibre, such as the microfibre knot resonator (MKR) [11], the microfibre loop resonator (MLR) [11] and the microfibre coil resonator (MCR) [12]. A microfibre coil resonator (MCR) has also been widely used in sensor and communications systems. However, very little research has been reported regarding lasing applications to date. An MCR has the inherent potential to provide a comb filter-like spectral characteristic in a fibre

* Corresponding author at: Key Laboratory of In-fiber Integrated Optics of Ministry of Education, College of Science, Harbin Engineering University, Harbin 150001, China.

E-mail address: pengfei.wang@dit.ie (P. Wang). 


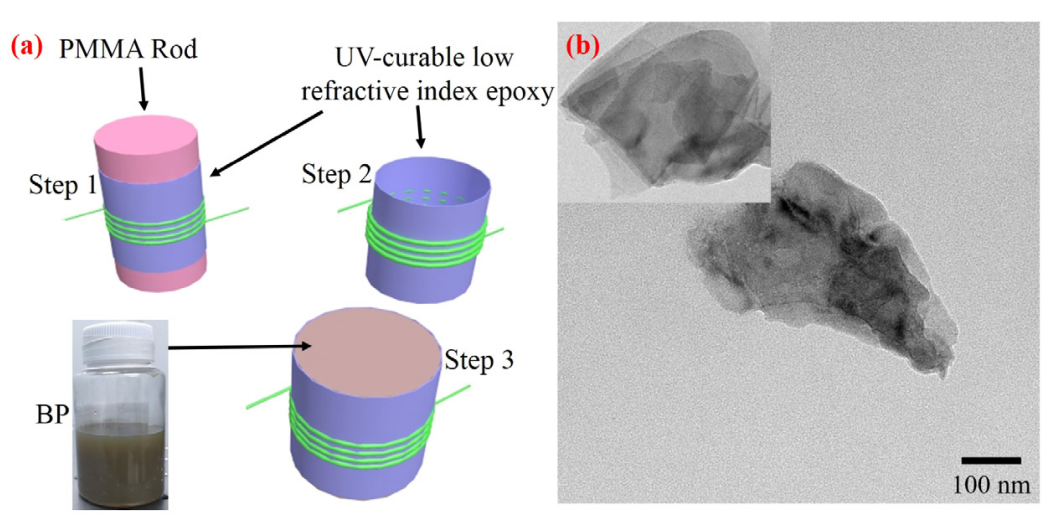

Fig. 1. (a) Schematic illustrations for the construction of the microfibre coil resonator incorporating black phosphorus (MCR-BP). (b) TEM image of the BP.

laser due to the presence of the whispering gallery mode (WGM) of the MCR [13], which make it possible to insert the MCR into the fibre laser to generate multi-wavelengths.

In this article, a novel device based on a microfibre coil resonator incorporating black phosphorus (MCR-BP) is described which is well suited to playing a key role as a low-cost discrete device comb filter whilst also providing the FWM level need to achieve a stable continuous wave multi-wavelength (CW) Tm-doped fibre laser. The slope efficiency of a thulium (Tm) doped fibre enables laser gain fibres to provide a high quantum efficiency and thus a high gain per unit length around the $2 \mu \mathrm{m}$ wavelength region. The few-layer black phosphorus was deposited onto the microfibre coil resonator to form the novel MCR-BP device. The MCR-BP device was inserted into the all-fibre laser cavity. Stable lasing has been achieved with twelve individual peaks with a $0.54 \mathrm{~nm}$ channel spacing, with every peak having an extinction ratio of at least $40 \mathrm{~dB}$.

\section{Experiment}

A microfibre coil resonator consisting of a 4-turn microcoil incorporating a fluidic channel was fabricated. A microfibre (SMF-28) with $2.24 \mu \mathrm{m}$ waist diameter was fabricated using the fused taper method [10]. The microfibre was wrapped on a $1 \mathrm{~mm}$-diameter PMMA rod (Goodfellow, ME307901) covered with UV-curable low refractive index epoxy (Luvantix, PC-373-LD) and fully cured using a UV lamp (Lightningcure, LC8). In order to avoid unnecessary loss, the MCR was additionally packaged using the same low refractive index epoxy and fully cured using the UV lamp. The MCR was subsequently soaked in an acetone solution to dissolve the PMMA rod which resulted in the MCR device forming a hollow cylindrical structure. Finally, a black phosphorus aqueous solution $(0.2 \mathrm{mg} / \mathrm{ml})$ was injected into the hollowed MCR and allowed to dry for several hours at room temperature. The preparation process is illustrated in full in Fig. 1(a) and the few-layer black phosphorus (XF 207, 7723-14-0, 102046) used in the experiment was prepared using the liquid-phase exfoliation method. In order to characteristic the layered structure of BP, Fig. 1(b) shows the TEM image of the BP.

To investigate the spectral characteristics of the resonator, a super continuum light source (YSL SC-series) was connected to one end of the MCR-BP device as a broadband light input with a range of 470-2400 nm and an OSA (optical spectrum analyzer: YOKOGAWA, AQ-6370C) was connected to the other end to measure the output spectral response. A typical transmission spectrum is shown in Fig. 2. The resulting Free Spectral Range (FSR) was measured to be $0.54 \mathrm{~nm}$. The microcoil was selected to include four turns in the experiment because it was determined that four turns ensured that the microfibre had a sufficiently large interaction length with the black phosphorus, whilst maintaining the good long-term mechanical stability for the MCR-BP structure, since it was found that having more than four turns may result in mechanical instability for the MCR.

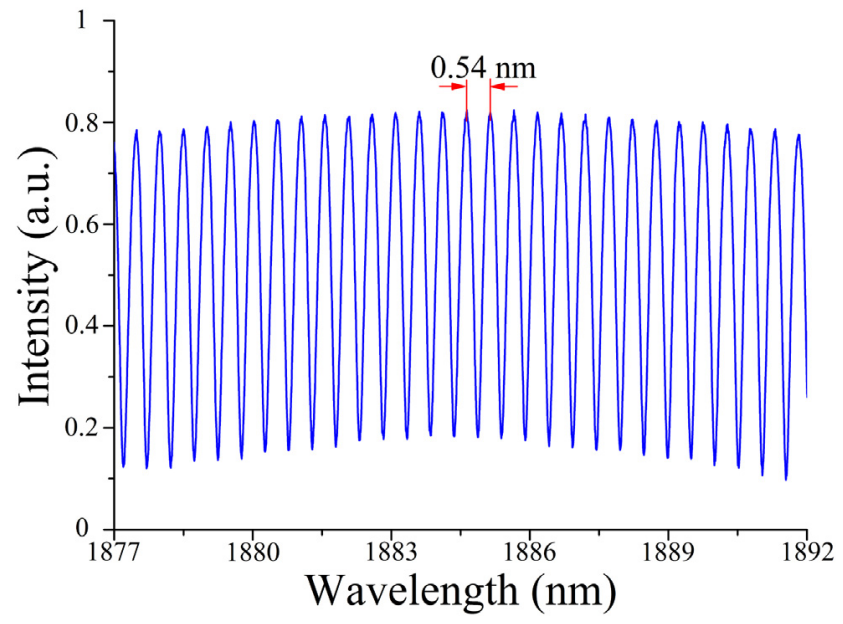

Fig. 2. Transmission spectrum of the MCR-BP device.

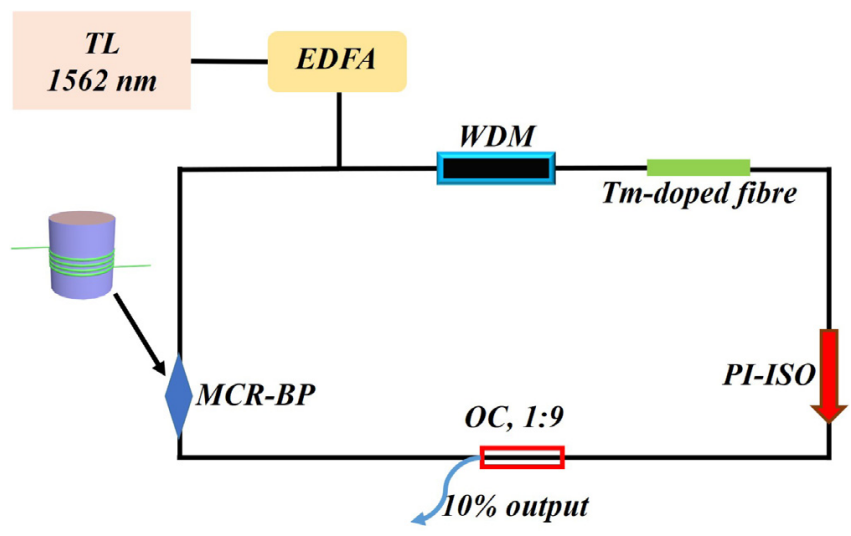

Fig. 3. Schematic of the experimental setup.

The complete experimental setup of the MCR laser system is shown in Fig. 3. A tunable laser (Santec TSI-710) centred at $1562 \mathrm{~nm}$ and an EDFA (MC-EDFA-230) was used as the pump source with a maximum power value of $700 \mathrm{~mW}$. A 1570/1980 $\mathrm{nm}$ wavelength division multiplexer (WDM, MCWDM-15/19) was inserted into the fibre laser cavity to pump the gain medium which consists of a $5 \mathrm{~m}$ length of Tm-doped fibre (Nufern SM-TSF-9/125). A polarization independent isolator (PI-ISO, MCI-1980) was used to maintain a unidirectional linear lasing. A 10/90 optical coupler (OC, 1980-FBT-C) was connected with the PI-ISO to measure the output spectrum using the OSA (YOKOGAWA, AQ-6370C). 


\section{Results and discussion}

In order to confirm that the MCR-BP device can be used for generating a stable multi-wavelength laser in the entire system, three cases were considered in the following experimental configurations: (1) no MCR used in the laser system; (2) MCR but without BP used; and (3) MCR with BP used. For the first case, the lasing output spectrum was recorded without an MCR used in the experiments. No stable multi-wavelength emission was observed in this case. However, a small number of unstable emissions were observed whose generation appeared erratic and variable when the pump power was varied from 0 to $700 \mathrm{~mW}$. A series of typical unstable spectra with the increasing time can be observed in Fig. 4(a) when the pump power was set to $200 \mathrm{~mW}$. A number of emissions in the vicinity of $1915 \mathrm{~nm}$ were obtained. The phenomenon indicated the clear instability of the output spectrum in the laser system. This result also indicates that strong mode-competition exists in the fibre laser.

For the second case, an MCR without the BP was subsequently inserted into the existing ring cavity. The MCR was fabricated on the same scale as the MCR-BP in order to minimize experimental error. This should result in the appearance of a comb-like filter being imposed on the cavity. The spectra obtained in the second case were recorded for 2-h interval and shown in Fig. 4(b), which indicated that the MCR was responsible for the generation of the comb-like filter laser output. The centre wavelength moved from $1915 \mathrm{~nm}$ (shown in Fig. 4(a)) to $1878 \mathrm{~nm}$ in Fig. 4(b), which can be largely attributed to the insertion loss when introducing the MCR. However, the power variation of the spectra can also be seen clearly in Fig. 4(b). The number of wavelengths clearly varied with increasing time, which indicated that the long-term stability is not satisfactory. The result indicates that strong mode-competition still exists in the lasing system with MCR but without BP.

Finally, the MCR-BP device was placed into the laser cavity. A single strong emission peak was observed centred at $1882.5 \mathrm{~nm}$ when the pump power was $116 \mathrm{~mW}$, which was evidence that the lasing threshold of the laser cavity had been reached. As the pump power was steadily increased, more peaks were observed. At a pumping power of $427 \mathrm{~mW}$, an output spectrum comprising twelve individual peaks was observed whose value ranged from $1879 \mathrm{~nm}$ to $1886 \mathrm{~nm}$ (shown in Fig. 5(a)). The wavelength interval between the peaks corresponds to a channel spacing of $0.54 \mathrm{~nm}$, while all twelve peaks exhibit an excellent extinction ratio (amplitude) of over $40 \mathrm{~dB}$. The peaks observed in this range were very uniform with an observed intensity fluctuation within $1.5 \mathrm{~dB}$ and the 3-dB linewidth of each channel was difficult to measure as they appear to be less than the spectral resolution $(0.05 \mathrm{~nm})$ of the OSA. The excellent uniform spectrum can be attributed to the power redistribution effect [14] of the FWM effect caused by the high nonlinearity of a few-layer black phosphorus. In order to further increase the number of the emission peaks, the following possible method could be pursued: minimize the cavity loss by reducing the length of the laser cavity and the insertion loss of each device in the ring cavity [15]. It is worth noting that the spectral channel spacing is dependent on the FSR of the MCR-BP device, which is associated with the thickness of the PMMA rod of the MCR [13]. Therefore, altering the channel spacing at the fabrication is a relatively simple task due to the ease of fabrication of the MCR.

A digital storage oscilloscope (DSO) (Tektronix MDO4054-6) was used to monitor the temporal characteristics of the 12-wavelength device. The result is shown in Fig. 5(b) and was obtained using a photodetector and amplifier (MC-PD-05) in conjunction with the DSO. The oscilloscope trace clearly shows that there was absolutely no pulselike behaviour observed in the output over a recording interval of $1000 \mu$ s confirming that the laser system operates as a Continuous Wave (CW) source.

A key issue in understanding why CW operation is achieved is whether black phosphorus has sufficient saturation absorption capacity to generate switched or mode-locked pulses, as there is no evidence that indicates that pulses which would characterize this behaviour existing
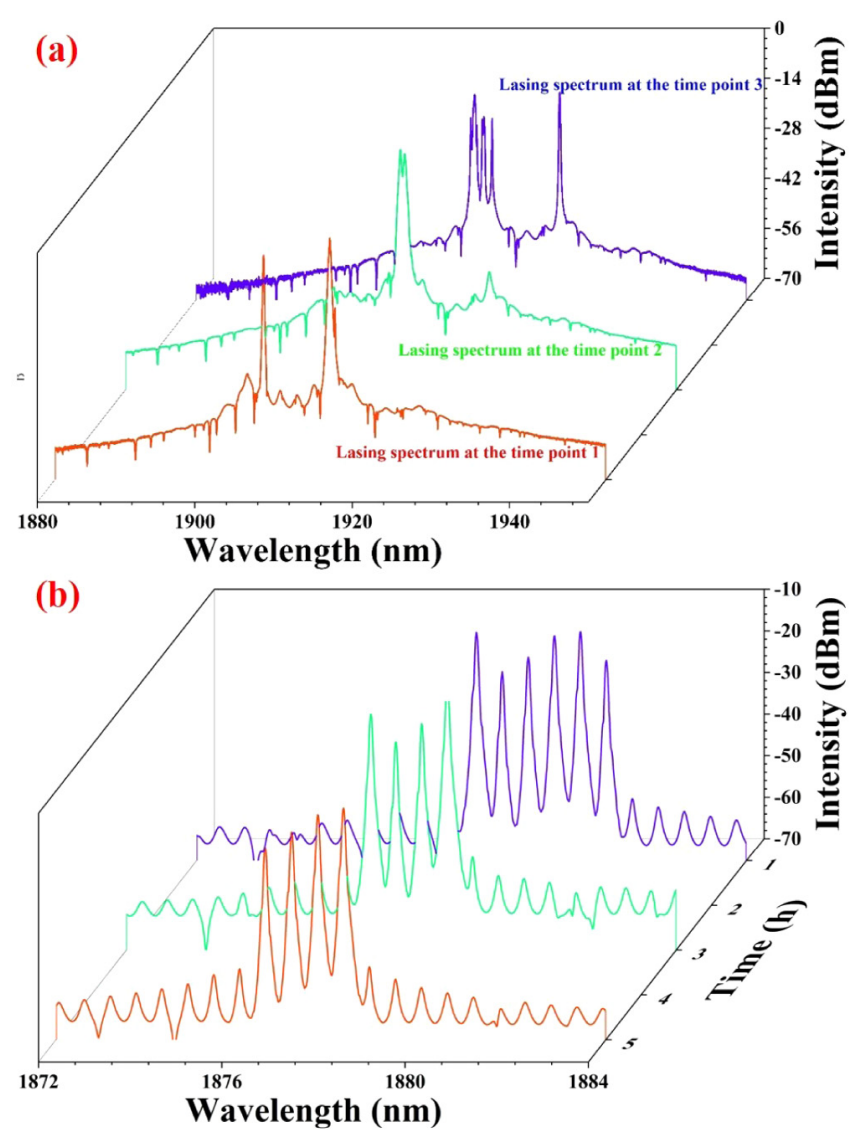

Fig. 4. (a) Spectra without an MCR in the cavity; (b) Spectra with an MCR only in the cavity.

in the results of Fig. 5(b). It is known that the generation of switched and mode-locked pulses needs a strict balance of the numbers of factors in the laser cavity such as nonlinearity and dispersion [16]. The most likely reason for the absence of pulses being observed in the output of the Tm-doped fibre laser, is that the configuration used does not match the balance conditions for switched or mode-locked operation.

In order to confirm the stability of the CW Tm-doped fibre laser, the $\mathrm{Tm}$-doped laser was operated continuously at room temperature with the source power fixed at $427 \mathrm{~mW}$ for more than $10 \mathrm{~h}$, and the longterm spectra were observed and recorded on a 2 -h interval. The results are shown in Fig. 6. As expected, no obvious fluctuations were observed which can be explained by the self-stabilizing effect derived from the FWM of the black phosphorus [8]. From the data in Fig. 6 which shows the long-term spectra, the largest observed power fluctuation across all the peaks is less than $1.6 \mathrm{~dB}$.

\section{Conclusion}

Experimental results have shown that a stable twelve-wavelength continuous Tm-doped fibre laser based on a novel device with a microfibre coil resonator incorporating black phosphorus has been successfully fabricated and demonstrated. The numerous observed stable output channels benefited from the interaction between the comb filter effect of the microfibre coil resonator and the mode competition suppression effect caused by the high nonlinearity of the black phosphorus coating. Twelve individual output peaks were obtained with a $0.54 \mathrm{~nm}$ channel spacing, a greater than $40 \mathrm{~dB}$ extinction ratio and a power fluctuation between channels of less than $1.6 \mathrm{~dB}$. The laser exhibited excellent intensity flatness of the 12 peaks and a 3-dB linewidth of less than $0.05 \mathrm{~nm}$ (less than the resolution of the OSA). The novel device based 

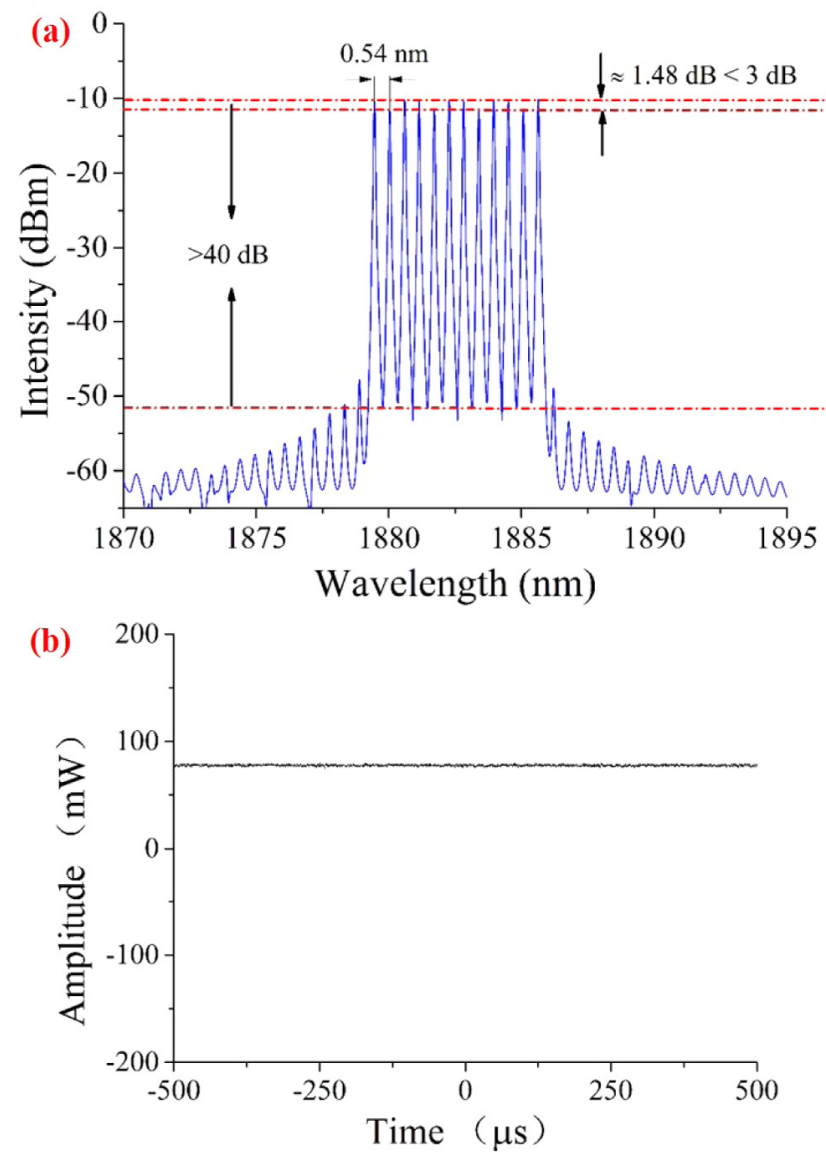

Fig. 5. (a) Multiwavelength output spectrum at the pump power of $427 \mathrm{~mW}$; (b) Oscilloscope trace of the measured temporal characteristic.

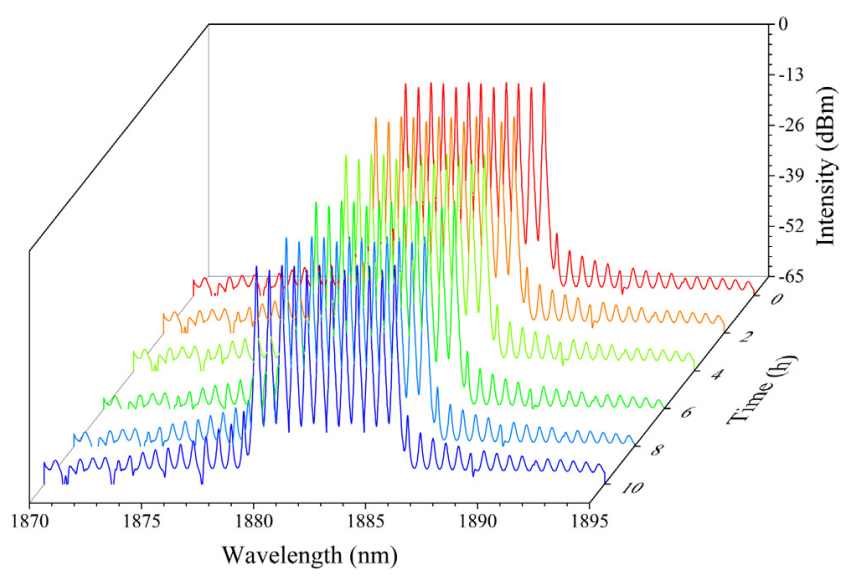

Fig. 6. Long term spectral variation measured at a 2-h interval over $10 \mathrm{~h}$.

on the microfibre coil resonator incorporating black phosphorus has proved that it represents an excellent opportunity to realize a stable and flat multi-wavelength fibre laser. The compact size and easy fabrication of the MCR-BP device provide a new solution to make a novel fibre laser device that is low loss, which is more compact with better output capability compared to existing state of the art devices.

\section{Funding}

The work was supported by the National Natural Science Foundation of China (NSFC) under grant 61575050; National Key R\&D Program of China under grant 2016YFE0126500; Key Program for Natural Science Foundation of Heilongjiang Province of China under grant ZD2016012; the Open Fund of the State Key Laboratory on Integrated Optoelectronics (Grant no: IOSKL2016KF03), this work was also supported by the 111 project to the Harbin Engineering University (B13015) and the fundamental research funds for the central universities.

\section{References}

[1] N. Park, P.F. Wysocki, 24-line multiwavelength operation of erbium-doped fiberring laser, IEEE Photonics Technol. Lett. 8 (1996) 1459-1461, http://dx.doi.org/ $10.1109 / 68.541549$.

[2] X. Ma, S. Luo, D. Chen, Switchable and tunable thulium-doped fiber laser incorporating a Sagnac loop mirror, Appl. Opt. 53 (2014) 4382-4385, http://dx.doi.org/ 10.1364/AO.53.004382.

[3] X. Feng, H.-y. Tam, P. Wai, Stable and uniform multiwavelength erbium-doped fiber laser using nonlinear polarization rotation, Opt. Express 14 (2006) 8205-8210, http://dx.doi.org/10.1364/OE.14.008205.

[4] H. Ahmad, S. Shahi, S. Harun, Multi-wavelength laser generation with bismuthbased erbium-doped fiber, Opt. Express 17 (2009) 203-207, http://dx.doi.org/10. 1364/OE.17.000203.

[5] G. Sun, D.S. Moon, A. Lin, W.-T. Han, Y. Chung, Tunable multiwavelength fiber laser using a comb filter based on erbium-ytterbium co-doped polarization maintaining fiber loop mirror, Opt. Express 16 (2008) 3652-3658, http://dx.doi. org/10.1364/OE.17.000203.

[6] B.-A. Yu, J. Kwon, S. Chung, S.-W. Seo, B. Lee, Multiwavelength-switchable SOAfibre ring laser using sampled Hi-Bi fibre grating, Electron. Lett. 39 (2003) 649-650, http://dx.doi.org/10.1049/el:20030435.

[7] E. Hendry, P.J. Hale, J. Moger, A. Savchenko, S. Mikhailov, Coherent nonlinear optical response of graphene, Phys. Rev. Lett. 105 (2010) 097401, http://dx.doi. org/10.1103/PhysRevLett.105.097401.

[8] S. Lu, L. Miao, Z. Guo, X. Qi, C. Zhao, H. Zhang, S. Wen, D. Tang, D. Fan, Broadband nonlinear optical response in multi-layer black phosphorus: an emerging infrared and mid-infrared optical material, Opt. Express 23 (2015) 11183-11194, http://dx.doi.org/10.1364/OE.23.011183.

[9] J. Sotor, G. Sobon, M. Kowalczyk, W. Macherzynski, P. Paletko, K.M. Abramski, Ultrafast thulium-doped fiber laser mode locked with black phosphorus, Opt. Lett. 40 (2015) 3885-3888, http://dx.doi.org/10.1364/OL.40.003885.

[10] L. Tong, F. Zi, X. Guo, J. Lou, Optical microfibers and nanofibers: A tutorial, Opt. Commun. 285 (2012) 4641-4647, http://dx.doi.org/10.1016/j.optcom.2012.07. 068.

[11] L. Tong, R.R. Gattass, J.B. Ashcom, S. He, J. Lou, M. Shen, I. Maxwell, E. Mazur, Subwavelength-diameter silica wires for low-loss optical wave guiding, Nature 426 (2003) 816, http://dx.doi.org/10.1038/nature02193.

[12] F. Xu, P. Horak, G. Brambilla, Optical microfiber coil resonator refractometric sensor, Opt. Express 15 (2007) 7888-7893, http://dx.doi.org/10.1364/oe.15.007888.

[13] M.-S. Yoon, H.-J. Kim, G. Brambilla, Y.-G. Han, Development of a small-size embedded optical microfiber coil resonator with high Q, J. Korean Phys. Soc. 61 (2012) 1381-1385, http://dx.doi.org/10.3938/jkps.61.1381.

[14] X. Liu, C. Lu, Self-stabilizing effect of four-wave mixing and its applications on multiwavelength erbium-doped fiber lasers, IEEE Photonics Technol. Lett. 17 (2005) 2541-2543, http://dx.doi.org/10.1109/lpt.2005.858075.

[15] Z. Luo, M. Zhou, Z. Cai, C. Ye, J. Weng, G. Huang, H. Xu, Graphene-assisted multiwavelength erbium-doped fiber ring laser, IEEE Photonics Technol. Lett. 23 (2011) 501-503, http://dx.doi.org/10.1109/lpt.2011.2112762.

[16] C. Zhao, H. Zhang, X. Qi, Y. Chen, Z. Wang, S. Wen, D. Tang, Ultra-short pulse generation by a topological insulator based saturable absorber, Appl. Phys. Lett. 101 (2012) 211106, http://dx.doi.org/10.1063/1.4767919. 